\title{
The Simulation Study of Auto-disturbance Rejection Controller on Unmanned Tracked Vehicle Steering
}

\author{
Lin Yao ${ }^{1, a}$, Hongwei Yang $^{2}$ and Hanqing Zhao ${ }^{3}$ \\ ${ }^{1,2,3}$ Changxindian Town NO.21, Fengtai District ,Beijing \\ aYLttkxwawj@163.com
}

Keywords: Auto-disturbance Rejection Controller; Unmanned Vehicles; Steering Control; System Smulation

\begin{abstract}
Motion control of autonomous vehicles is a key technology in the research field of unmanned vehicles and is of great importance in the operative part of autonomous driving. This paper regards a certain type of crawler as the controlled object. First of all, the paper establishes a vehicle steering kinetic model for it by using approximate mechanism modeling, and simulates vehicle steering control by adopting auto-disturbance rejection controller and simulink. The simulation result shows that auto-disturbance rejection controller can realize signal tracking effectively, also have shorter steering response time and small overshoot, and it is more accurate by comparing with the traditional PID control method.
\end{abstract}

\section{Introduction}

With the constant development and progress of current technology, the degree of automation and intelligence is increasingly high in routine production life. Unmanned vehicles are national research objects. Generally speaking, control of unmanned vehicles can be divided into three parts, including environment perception, programmed decision-making and accuracy control[1]. Just like response mechanism on external objects, this paper mainly carries out the simulation research on steering control problems of autonomous vehicles.

\section{Problem Elaboration}

Steering Control Problems of Autonomous Crawlers

Steering control of unmanned vehicles refers to study how to control steering mechanism of vehicles and make actual motion trails of vehicles have the minimum with the planned expected routes, in the condition of giving expected motion trails or planning target points[2]. This is also the core problem in motion control of unmanned vehicles.

On the other hand, steering of crawlers differs a lot from wheeled vehicles[3]. For example, with different steering mechanism, diverse steering resistance and the steering controllability, crawlers have larger difficulty. It is necessary to study steering problems of crawlers more carefully. This will have larger significance on dual-use and unmanned technology.

Explanation of Control Algorithm

The industrial controller represented by PID receives the extensive application and still has occupied a leading position until now[4]. However, steering control of vehicles has certain 
nonlinearity, and control system receives complicated internal and external disturbance and has higher uncertainty, so the control effect of traditional PID control method is not good. Thus, this paper selects the auto-disturbance rejection controller proposed by a researcher Han Jingqing. This is the inheritance and development of thinking essence for PID control method. Its core idea is to estimate and compensate system unknown disturbance and unknown model as "total disturbance" of the system. This promotes control quality and precision in essence[5] and also is more suitable for control problems studied by this paper.

\section{Steering Control Modeling of Crawlers}

This paper only considers two aspects involved by vehicle steering, including the driving force and braking force. The driving force is mainly provided by the engine, while braking power sources are relatively complicated. Here researchers should give priority to the frictional resistance between continuous tracks and the ground.

For this model, researchers provide the following hypotheses:

1) Geometrically, vehicles are symmetric in longitudinal plate and horizontal plane. The projection of centroid on the horizontal plane coincides with plane geometric center of vehicles;

2) Vehicles steer evenly at low speed on the horizontal ground and ignore centrifugal influences;

3) Continuous tracks are uniform soft belts and lose sight of width influences;

4) When steering, driving resistance coefficient is equal to straight driving;

5) Vehicles only conduct plane motion with three degrees of freedom.

For the law of rotation for rigid body to roll dead axle, researchers can conduct approximate mechanism modeling in line with the following formula.

$$
T_{z}-M_{r}=m R^{2} \ddot{\theta}
$$

Eq.1

In the formula, $T_{z}$ is the torque of capstan for steering vehicles, $M_{r}$ is steering drag torque for steering vehicles, $\mathrm{m}$ is the quality of crawlers, $\mathrm{R}$ is steering radius, and $\ddot{\theta}$ is the accelerated speed of steering angles.

Researchers adopt empirical formula of engine power in the reference [3], and drag torque formula in the reference [6], steering dynamic model of vehicles ultimately can be obtained:

$$
\left(2020.5684-\frac{220963.86}{n_{e}}\right) i_{1} \eta-\frac{m g \mu L}{8}\left[1+\left(\sqrt{1+a_{2}^{2}}-\frac{a_{2}^{2}}{2} \ln \frac{\sqrt{1+a_{2}^{2}}+1}{\sqrt{1+a_{2}^{2}}-1}\right)\right]=m R^{2} \ddot{\theta}
$$

In the formula, $\mathrm{m}$ is vehicle quality, $\mathrm{g}$ is acceleration of gravity, $\mu$ is ground steering resistance coefficient, $\mathrm{L}$ is field length of continuous tracks, and steering horizontal related offset a is redefined, and $a=A /(L / 2), \mathrm{i}$ is total drive ratio for a certain gear, and $\eta$ is efficiency, $n_{e}$ is engine speed.

\section{Auto-disturbance Rejection Controller (ADRC)}

The block diagram of ADRC is shown in Fig. 1: 


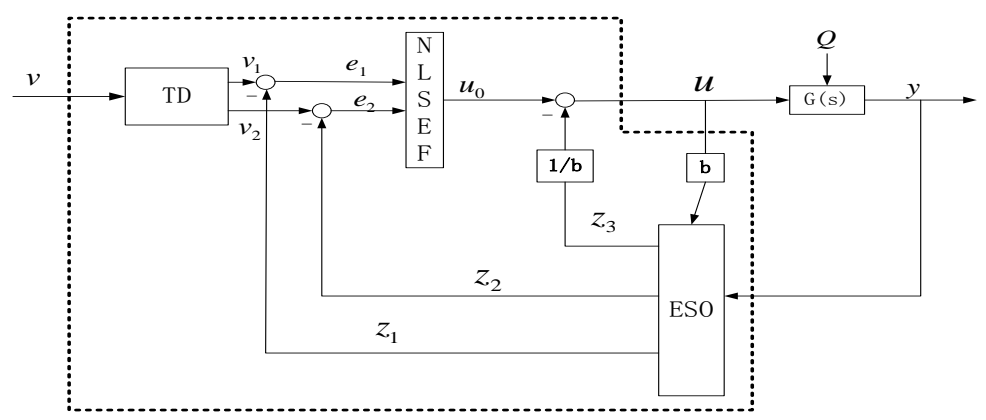

Fig. 1

It mainly composes of three parts: tracking differentiator, extended state observer and nonlinear state error feedback. Its function can be briefly indicated that the tracking differentiator (TD) can obtain tracking signal v1 of original input signal and approximate differential signal v2 of original input signal, so as to arrange transient process systematically. Extended state observer (ESO) can observe system state and action of controlled objects in internal disturbance and external disturbance in real time. Then, this action is compensated for the controller to realize linear feedback of nonlinear system. Non-linear error feedback is to conduct appropriate combination for signal generated by tracking differentiator and extended state observer through a nonlinear function to generate the controlled quantity and transport to controlled objects.

Typical second order auto-disturbance rejection controller is as follows:

$$
\left\{\begin{array}{c}
f h=\operatorname{fhan}\left(v_{1}-v, v_{2}, r_{0}, h\right) \\
v_{1}=v_{1}+h v_{2} \\
v_{2}=v_{2}+h f h \\
e=z_{1}-y, f e=f a l(e, 0.5, h), f e_{1}=f a l(e, 0.25, h) \\
z_{1}=z_{1}+h\left(z_{2}-\beta_{01} e\right) \\
z_{2}=z_{2}+h\left(z_{3}-\beta_{02} f e+u\right) \\
z_{3}=z_{3}+h\left(-\beta_{03} f e_{1}\right) \\
e_{1}=v_{1}-z_{1}, e_{2}=v_{2}-z_{2} \\
u=-\operatorname{fhan}\left(e_{1}, c e_{2}, r, h\right)-z_{3}
\end{array}\right.
$$

In the algorithm of $\mathbf{f a l}(\mathbf{x}, \boldsymbol{\alpha}, \mathbf{d})$ and $\mathbf{f h a n}\left(x_{1}, x_{2}, \mathbf{r}, \mathbf{h}\right)$, the definition is as follows:

$$
f a l(x, \alpha, d)=\left\{\begin{array}{c}
x / d^{1-\alpha},|x| \leq d \\
|x|^{\alpha} \operatorname{sign}(x),|x|>d
\end{array}\right.
$$

and 


$$
\left\{\begin{array}{c}
d=r h^{2}, a_{0}=h x_{2}, y=x_{1}+a_{0} \\
a_{1}=\sqrt{d(d+8|y|)} \\
a_{2}=a_{0}+\operatorname{sign}(y)\left(a_{1}-d\right) / 2 \\
s_{y}=(\operatorname{sign}(y+d)-\operatorname{sign}(y-d)) / 2 \\
a=\left(a_{0}+y-a_{2}\right) s_{y}+a_{2} \\
s_{a}=(\operatorname{sign}(a+d)-\operatorname{sign}(a-d)) / 2 \\
\text { fhan }=-r(a / d-\operatorname{sign}(a)) s_{a}-r \operatorname{sign}(a)
\end{array}\right.
$$

Here, $r_{\mathbf{0}}$ is used for adjusting speed of transient process and is mainly decided by system bearing capacity; $\beta_{01}, \beta_{02}$ and $\beta_{03}$ are determined by adoptive time step. Even if objects are different, and time step is the same, it also can adopt the same parameter value. $r$ is controlled quantity gain. Generally speaking, it dispenses with regulation to a certain extent; $\mathrm{c}$ is damping

coefficient; is precision factor; $b_{\mathbf{0}}$ is compensating factor. The latter three ones need in line with parameters of object regulation in real system.

\section{Simulation Example and Result Analysis}

Assuming that basic parameter of crawlers in our actual control is as follows:

Vehicle weight $\mathrm{m}=36000 \mathrm{~kg}$, acceleration of gravity $\mathrm{g}=9.8 \mathrm{~m} / \mathrm{s}^{2}$, vehicle steering radius $\mathrm{R}=\mathrm{B} / 2=1.31 \mathrm{~m}$, let vehicles conduct first gear pivot steering, transmission ratio of first gear is $i_{1}=28$, and overall efficiency $\eta=0.7$.

Then, researchers adopt classic PID algorithm and auto-disturbance rejection controller, respectively to conduct simulation tracking on step signal, square signal and sinusoidal signal.

Simulink simulation procedure charts of classic PID algorithm and auto-disturbance rejection controller are shown in Fig. 2 and Fig. 3, respectively:

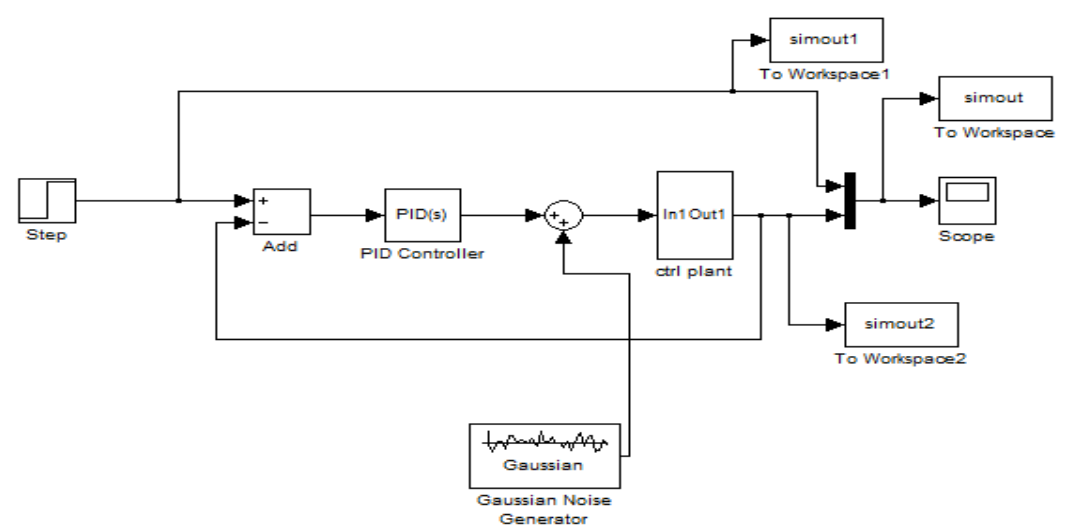

Fig. 2 PID Simulation Procedure Chart 


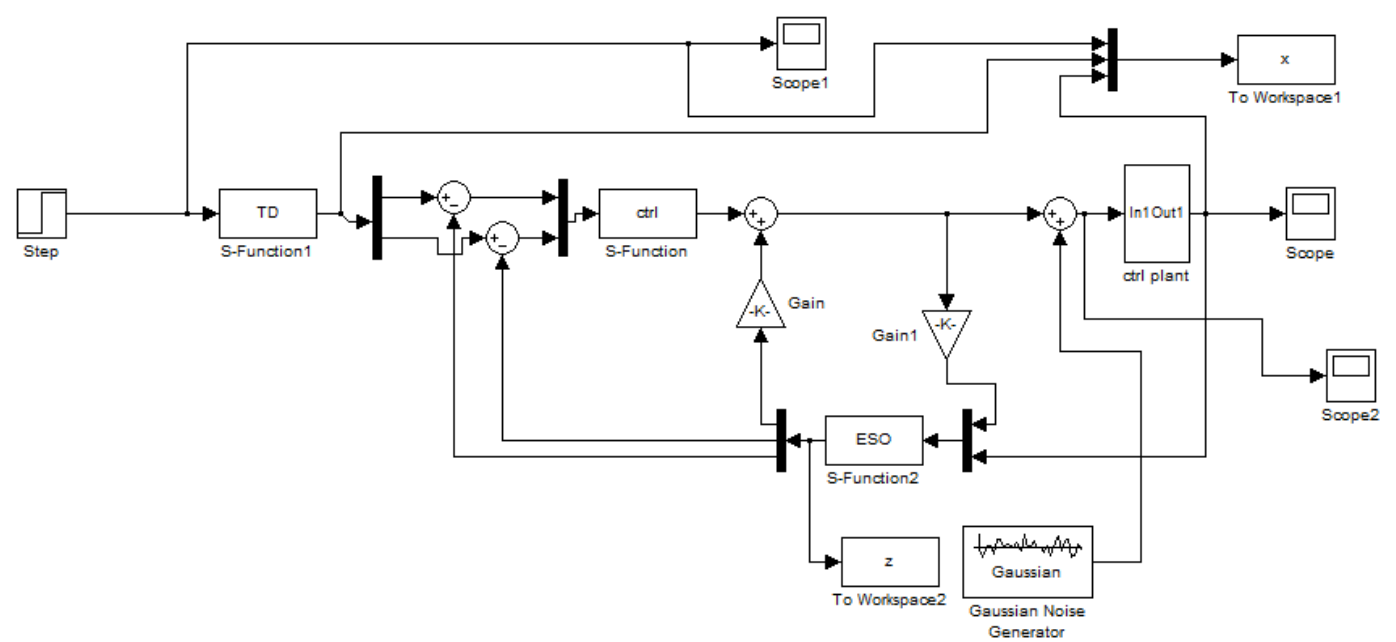

Fig. 3 ADRC Simulation Procedure Chart

Simulation results of two methods are shown in Fig. 4(the following realized result is original signal, and dotted line is tracking signal):

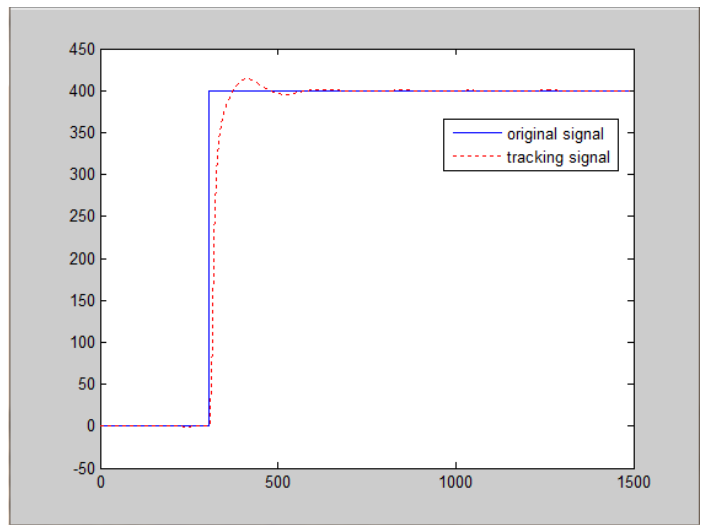

PID

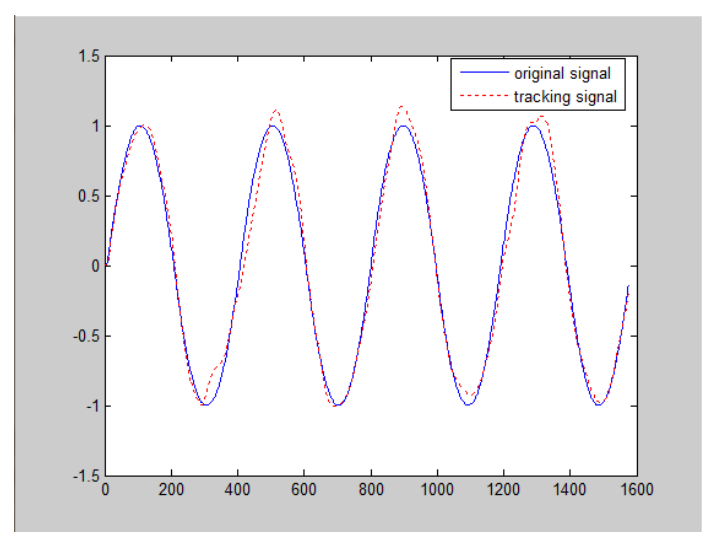

PID

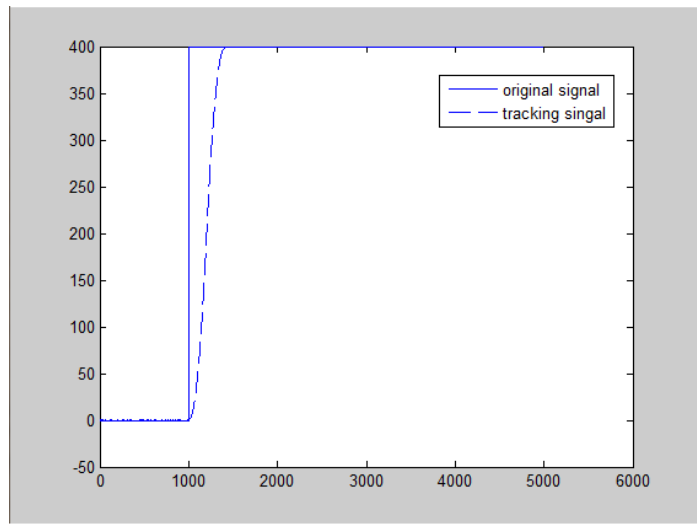

ADRC

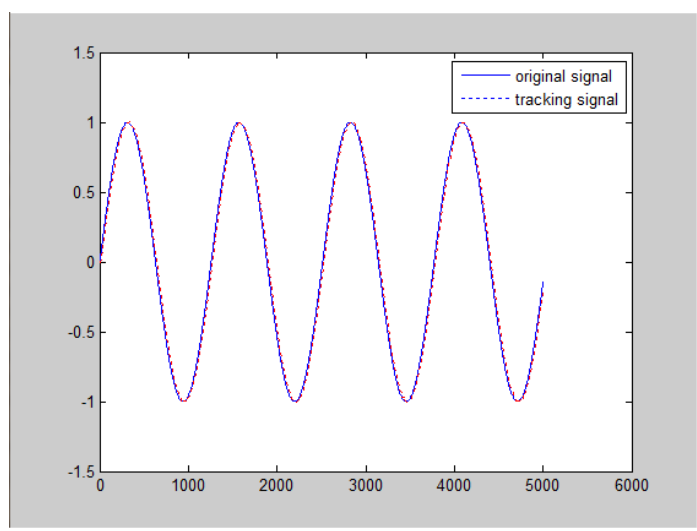

ADRC 


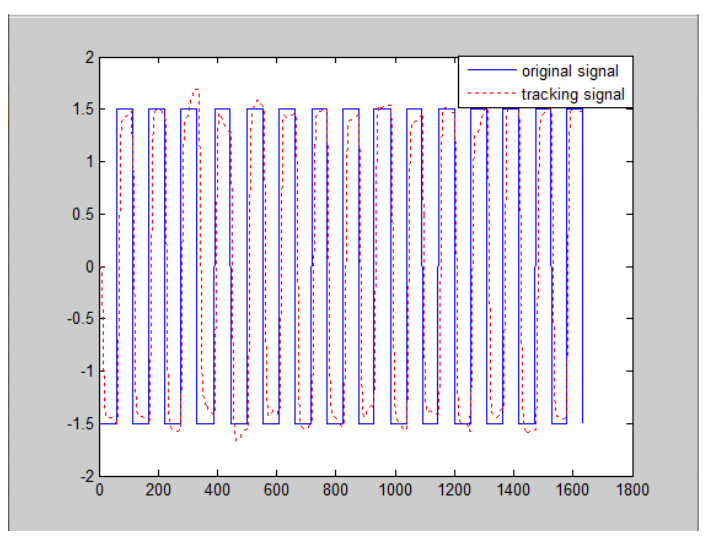

PID

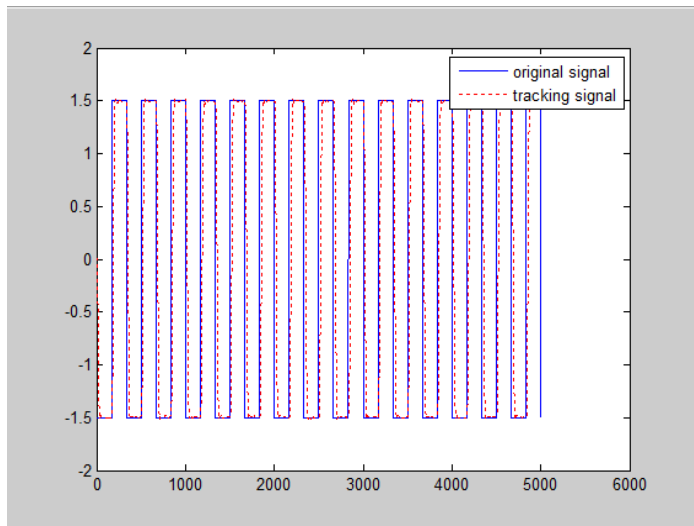

ADRC

Fig. 4 Simulation Result

The above-mentioned three figures are tracking results of step, sine and square signals in classic PID algorithm and auto-disturbance rejection controller. In PID, $k_{p}, k_{i}$, and $k_{d}$ parameters are set up as 8, 9 and 10. In ADRC, larger influences of r, c, and $b_{0}$ parameters are set up as $100,0.5$, 0.05 and 1 .

On the one hand, it can be observed obviously that objects controlled by ADRC have better tracking precision and smaller overshoot. Tracking time superiority in step signal is worse, but has stronger stability. In a short, its effect is better than PID. On the other hand, in regulation process, for different input signals, three parameters must be modified repeatedly and thus can reach a good effect. A set of parameters in this paper has relatively good tracking effect for these three signals. Moreover, before and after adding disturbance, tracking effect has larger difference. After confirming a parameter in ADRC algorithm, it has stable tracking effect on various input signals and before and after adding disturbance.

\section{Conclusions}

This paper regards unmanned crawlers as research objects, conducts simulation research on steering control problems, designs auto-disturbance-rejection controller to track given signal instruction in accordance with steering model established by approximate mechanism, and also uses classic PID control algorithm to complete the same work to make a comparison. Simulation result shows that:

1. Auto-disturbance rejection controller can be used for controlling steering system of crawlers and have higher precision;

2. In restraining disturbance ability, auto-disturbance rejection controller is stronger than classic PID algorithm and also has better robustness;

Shortcomings mainly lie in adjusting parameters. Parameter quantity is larger and its adjustment is complicated. 


\section{References}

[1] Yu Xiangmin, Motion Control Technique of Autonomous Land Vehicles, Master's thesis of Armored Force Engineering Institute, 2013.

[2] Zhao Hanqing, the Learning Control Study on SVR Rarefaction and Autonomous Land Vehicles, Doctoral dissertation of National University of Defense Technology, 2008.

[3] Yan Qingdong, Zhang Liandi and Zhao Yuqin, Tank Structure and Design (volume two), Beijing Institute of Technology Press, 2007.

[4] Jiangping, Hao Jingyu and Zong Xiaoping, et al, Simulink Modeling Simulation of Auto-disturbance Rejection Controller, J. Control Theory and Application, 2010, 29(2),pp.0001-0005.

[5] Han Jingqing, Auto-disturbance Rejection Controller, J. Frontier Science, 2007.1(1), pp.24-31;

[6] Cheng Junwei, Gao Lianhua and Wang Hongyan et al., Steering Analysis of Crawlers, J. Journal of Ordnance Factory, 2007.28(9), pp. 1110-1115.

[7] Han Jingqing, From PID Technology to "Auto-disturbance Rejection Controller", J. Control Engineering, 2002.9(3), pp.0013-0018.

[8] Li Yiran, Zhou Jie and Zhan Xilan et al., the Study on Auto-disturbance Rejection Controller of Unmanned Vehicles' Steering System, J. Journal of Shanghai Normal University: natural science, 2010.39(2),pp.0156-0160.

[9] Han Jingqing, Auto-disturbance Rejection Controller, National Defense Industry Press, 2013.

[10] Yingjie Zhang, Chaodong Fan, Fangfang Zhao, et al, Parameter tuning of ADRC and its applicaton based on CCCSA, J. Nonlinear Dyn(2014)76, pp.1185-1194. 